\title{
A Long Reach Coherent m-QAM OFDM PON Downstream Transmission
}

\author{
Vyankatesh Maske ${ }^{1}$, Pawase Ramesh $\mathrm{S}^{1}$ and R.P.Labade ${ }^{1}$ \\ ${ }^{1}$ Electronics and Telecommunication Department \\ Amrutvahini college of Engineering, Sangamner, India \\ Email:vyanky24@gmail.com
}

\begin{abstract}
In this paper OFDM-PON downstream transmission is demonstrated over different length of fiber at Remote Node (RN) for different $m$-QAM mapped OFDM signal ( $m=4$ and16) transmission from the central office (CO) for data rates (10 Gbps) using coherent detection at the user end or optical network unit (ONU). Investigation is performed with different number of sub-carriers back to back optical signal to noise ratio (OSNR) along with transmitted and received constellation diagram for m-QAM mapped coherent OFDM downstream transmission at different speed over different transmission distance. Simulation results suggest the different length and data rate that can be used for different $m$-QAM mapped coherent detection OFDM downstream transmission and the proposed system may be implemented in Next Generation high speed PONs (NG-PONs).
\end{abstract}

Keyword: Optical OFDM, SMF, Coherent detection

\section{Introduction}

The concept of internet broadband services is marvelously growing. In the world of telecommunication, there are subscribers demand for greater speed and lesser cost broadband services. It is a challenge for researchers to provide a higher speed of these services at optimum cost. A typical communication system combining voice data, video, and other multimedia services requires faster transmission capacity and flexibility. Now a day we all are living in the era of "unambiguous connectivity" or "communication anywhere at any time when needed". Recently, the telecommunication world and its applications like GSM and GPRS ( $2 \mathrm{G}$ system) realized quite low data rates [1]. For 2G data rate is $56 \mathrm{~Kb} / \mathrm{s}$ which uses Global System for Mobile Communication (GSM) technology and for $2.75 \mathrm{G}$ data rate is $236.8 \mathrm{~Kb} / \mathrm{s}$ uses Enhance Data Rate for GSM Evolution (EDGE) technology [2]. In 3G system like WCDMA data rate up to $140 \mathrm{Mbp} / \mathrm{s}$ [2] are also not very satisfactory as it has a large number of subscribers using internet broadband services for a variety of applications such as banking, paying electricity bills, mobile dish TV recharges, video conferencing and social communication required fast broadband services.

One of the solution to meet massive expected demand is to improve communication system, evokes a new idea of optical Orthogonal Frequency Division Multiplexing (OFDM) with coherent detection, and is referred as coherent optical OFDM or COOFDM technique. The optical communication system has numerous advantages like as very high bandwidth, low attenuation loss, immune to electromagnetic induction (EMI) \& high flexibility. Coherent optical OFDM is integrated with quadrature phase shift keying (QPSK) which can provide a great spectral efficiency and significantly high data rate [3]. Nowadays using optical OFDM for long haul transmission has proven better than conventional single carrier modulation technique. Coherent optical OFDM is really promising solution to satisfy the huge demand of subscribers for next generation communication systems. Basically increasing the number of users will pose limitations on 
data communications i.e. speed is reduced due to increasing number of users; as a result, alarge high bandwidth is required. Thus, there is a possibility of Multiple User Interference (MUI) and Inter-Symbol Interference (ISI), hence system complexity increases. The coherent optical OFDM technique has an objective to eliminate this issue. The performance of optical OFDM system is characterized using the bit error rate (BER); however the general BER for optical communication is $10^{-9}$. In CO-OFDM system fiber nonlinearity, phase noise and chromatic dispersion prominently limit the performance of OFDM signal owing to large peak-to-average power ratio (PAPR). There has a vibrant research on coherent optical OFDM technique to improve tolerance towards fiber nonlinearity [4], transmissions distance in long haul communication system [5] and reduce PAPR.

Optical OFDM system using standard single-mode fiber (SMF) is the preeminent way to achieve linearity between input transmitter inverse Fast Fourier Transform (IFFT) and output receiver Fast Fourier Transform (FFT) using linear field modulation [6]. In this radio-frequency $(\mathrm{RF})$ subcarrier is map onto an optical carrier and transmitted over an optical link to facilitate the wireless transmission. At the receiver,light signal can either be detected directly or coherently. Based on this detection scheme optical OFDM is further classified in two categories, first direct detection optical OFDM is referred as DDOOFDM and second coherent optical OFDM is referred as CO-OFDM. In DDO-OFDM single sideband field modulation is used to compensate issue regarding chromatic dispersion (CD) [4]. The advantage of DDO-OFDM is simple receiver have low cost, but it needed more transmitted optical power and some of the optical frequencies must be unused result in reduced spectral efficiency [6]. In CO-OFDM system locally generated carrier signal and received signal is mixed while at the receiver local carrier signal is generated using a laser. The main drawback of this system is fiber nonlinearity which affects the sensitivity of CO-FDM system. Non-linearity in optical channel is reduced to acertainextent with thehelp ofVolterra model. In Volterra model reorthogonalization techniques in modified Gram-Schmidt method helps in reducing fiber non-linearity [7]. Thus, both systems invoke new research field in optical OFDM and particularly in DDOOFDM and CO-OFDM techniques to mitigate the disadvantages.

In this paper, we have investigated the performance of OFDM-PON downstream transmission using m-QAM mapped OFDM transmission $(\mathrm{m}=4,16,32$ and 64$)$ at the $\mathrm{CO}$ and coherent detection at the receiver end (ONUs). The m-QAM modulated OFDM transmissions at $\mathrm{CO}$ have a data rate of 10,20,30 and 40Gbps. The performance of downstream coherent detection m-QAM mapped OFDM-PON is investigated in terms of constellation diagrams, back to back OSNR, distance travelled by different number of subcarriers for m-QAM coherent OFDM and received optical power at different BERs at different data rate. At the ONUs, the downstream OFDM signal is detected by coherent detection. In this architecture, loop-back upstream scheme may be implemented to realize the colorless upstream operation $[10,11]$. The remaining paper is organized under following headings- generation and detection of Coherent Detection OFDM, Proposed Architecture, Results and Discussion and Conclusion. 


\section{Coherent Optical OFDM}

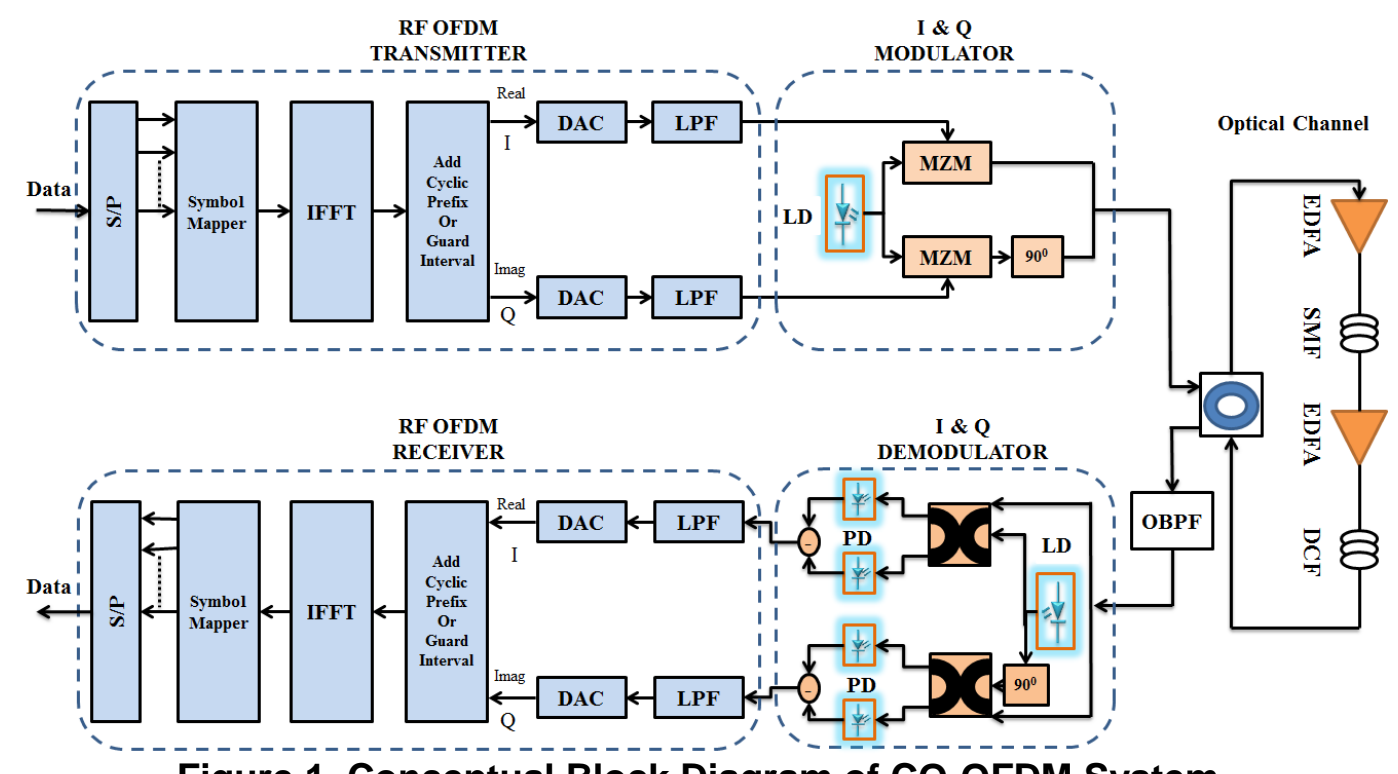

Figure 1. Conceptual Block Diagram of CO-OFDM System

\section{LD: Laser Diode PD: Photo Diode MZM: Mach-Zender Modulator DCF: Dispersion Compensation Fiber}

OFDM usually a standard coherent detection OFDM system is composed of five constructional blocks that are OFDM transmitter, RF to optical (RTO) up-converter, optical fiber transmission link, optical to RF (OTR) down-converter and coherent OFDM receiver. Figure 1 shows the schematic of m-QAM mapped coherent detection optical OFDM transmitter. First the PRBS data is fed to the QAM sequence generator that divides the PRBS data into two parallel sub-sequences that can be transmitted in two quadrature carriers by employing a serial to parallel converter. QAM sequence generator maps the input PRBS data into QAM symbols. The generated QAM sequence is fed to OFDM modulator. The OFDM modulator can have input data in different formats, like BPSK, QAM, 16-QAM etc. at the OFDM transmission, modulation and multiplexing is performed digitally by using inverse fast Fourier transform (IFFT). All the sub-carrier frequencies are mathematically orthogonal in the generated OFDM signal over each OFDM symbol period. The in phase and quadrature phase output from the OFDM modulator are filtered using low pass (LP) cosine roll-off filter. RTO block consists of a $\mathrm{CW}$ laser and two Mach-Zehnder modulators are employed to up-convert the RF OFDM data into the optical domain. Then the signal is propagated through optical fiber transmission link. Due to fiber impairments like attenuation, chromatic dispersion, nonlinearities etc. the optical signal degrades. In Optical channel,erbium-doped fiber amplifier (EDFA) is widely used for in-line amplification of the signal in optical communication. It provides high power transfer efficiency and independent from data rate. Optical channel use standard single mode fiber (SMF) with dispersion compensation fiber (DCF) to compensation of dispersion in the optical channel. DCF is having negative dispersion with the low positive slope. The coherent detection technique at receiver is capable of reproducing transmitted Inphase (I) and Quadrature (Q) signal at the receiver. The RF down conversion architecture of optical OFDM at receiver use two balanced pairsof optical hybrids with optical $90^{\circ}$ phase shifter to accomplish Inphase (I) and Quadrature (Q) detection as shown in Figure1. In RF demodulator block the exact reverse process of RF modulation was performed to recover original information or data signal. 


\section{Experimental Setup and Description}

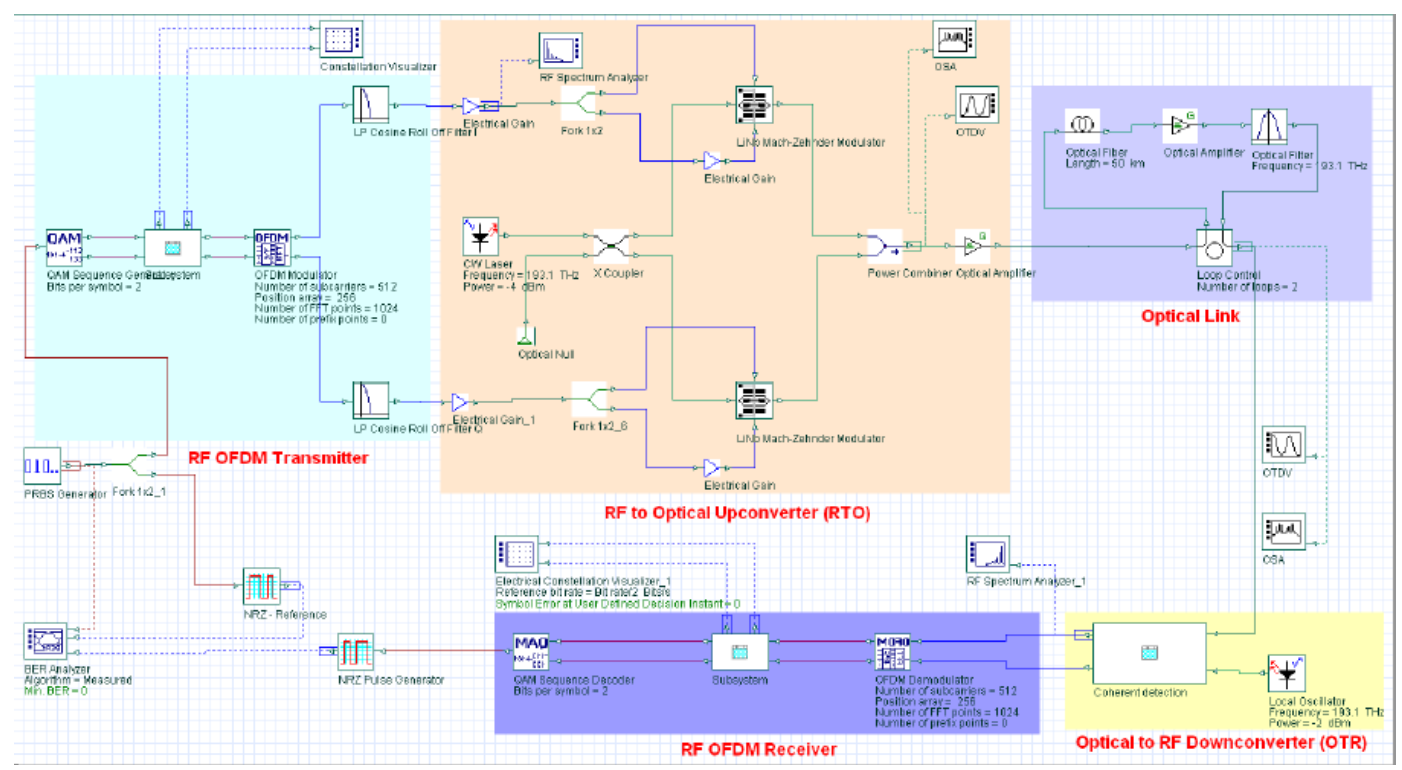

Figure 2. Simulation Set Up for Single User CO-OFDM for 4-QAM

Figure 2 show the proposed architecture of long reach OFDM-PON downstream transmission for different m-QAM mapped OFDM signal using coherent detection. At the CO, PRBS data of $2^{31}-1$ order is fed to QAM sequence generator with number of bit per symbol equal to $\mathrm{b}$ where $\mathrm{b}=2,4$, for $\mathrm{m}=4,16$, respectively. The m-QAM mapped QAM data is fed to OFDM modulator. The parameter prefix point indicated that how many points will be used in the guard period and set as 0 . After filtering using LP cosine roll off filter (cut-off frequency $=0.62 \times$ (bit rate), depth $=100 \mathrm{~dB}$, roll off factor $=0.2$ ) and amplification, In-phase and quadrature phase output of OFDM modulator is modulated on the optical carrier using two LiNb Mach-Zehnder modulators (MZM) with extinction ratio of $60 \mathrm{~dB}$ and a CW laser (center frequency $=193.1 \mathrm{THz}$ or $1550 \mathrm{~nm}$, line-width $=150$ $\mathrm{KHz}$ ). The output of two LiNb MZMs is combined using a power combiner therefore the output of power combiner is the optical OFDM signal. This optical OFDM signal is sent to RN. Table 1 shows the different parameters that are utilized for the simulation [14]. The RN is consists of a standard single mode fiber (SSMF) link, an optical amplifier and an optical filter. The optical OFDM signal received from CO is fed to SSMF (chromatic dispersion $(\mathrm{CD})=16 \mathrm{ps} / \mathrm{nm}-\mathrm{km}$, attenuation $=0.2 \mathrm{~dB} / \mathrm{km}$, effective area $=80(\mu \mathrm{m})^{\wedge} 2$ and dispersion slope $(\mathrm{DS})=0.075 \mathrm{ps} / \mathrm{nm}^{2}-\mathrm{km}$, respectively). The output signal from the SSMF is amplified using an optical amplifier (gain $=12 \mathrm{~dB}$, noise figure $=4 \mathrm{~dB}$ ), filtered by an optical filter (center frequency $=193.1 \mathrm{THz}$, bandwidth $=15 \mathrm{GHz}$, depth $=100 \mathrm{~dB}$, order $=2$ ) and after that sent to ONU for coherent detection. At the ONU, a local oscillator (frequency $=193.1 \mathrm{THz}$, power $=-2 \mathrm{dBm}$, line-width $=150 \mathrm{KHz}$ ) and balanced homodyne detection consisting of 4 couplers, a phase shifter and 4 photo-detectors $\left(\right.$ responsivity $=1 \mathrm{~A} / \mathrm{W}$, dark current $=10 \mathrm{nA}$, thermal power density $=100 \times 10^{-24} \mathrm{~W} / \mathrm{Hz}$ ) are utilized for the coherent detection of optical OFDM signal transmitted from the CO. After balanced homodyne detection of in-phase and quadrature phase components of optical OFDM; they are fed to OFDM demodulator. The output of OFDM demodulator is fed to the QAM sequence decoder and giving output. This output is utilized for BER calculations. In order to enhance the power budget and improve the dynamic range of the system, an optical amplifier is included at RN [15-16]. 
Table 1. Shows the Different Parameters That Are Utilized For the Simulation

\begin{tabular}{|c|c|}
\hline Parameter & SMF \\
\hline Dispersion & $16 \mathrm{ps} / \mathrm{nm} / \mathrm{km}$ \\
\hline Dispersion slope & $\begin{array}{c}-0.08 \\
\mathrm{ps} / \mathrm{nm}^{\wedge} 2 / \mathrm{km}\end{array}$ \\
\hline PMD coefficient & $0.2 \mathrm{ps} / \mathrm{km}$ \\
\hline Effective Area & $80 \mathrm{um}^{\wedge} 2$ \\
\hline Nonlinearity Coefficient & $2.6 \mathrm{e} 10^{\wedge}-20$ \\
\hline Attenuation & $0.2 \mathrm{~dB} / \mathrm{Km}$ \\
\hline
\end{tabular}

\section{Result and Discussion}

At the transmission block, both modulation and multiplexing are achieved digitally using an inverse fast Fourier transform (IFFT). The subcarrier frequencies are mathematically orthogonal over one OFDM symbol period. A CW laser and two MachZehnder modulators are used to up-convert the RF data to the optical domain. The signal is then propagated through the optical link and becomes degraded due to fiber impairments. A coherent receiver with a local oscillator is used to down-convert the data to the RF domain, and finally data is demodulated and sent to the detector and decoder for BER measurements. Transmitted signal constellation diagram for 4QAM at the RF OFDM transmitter is shown in figure 3.

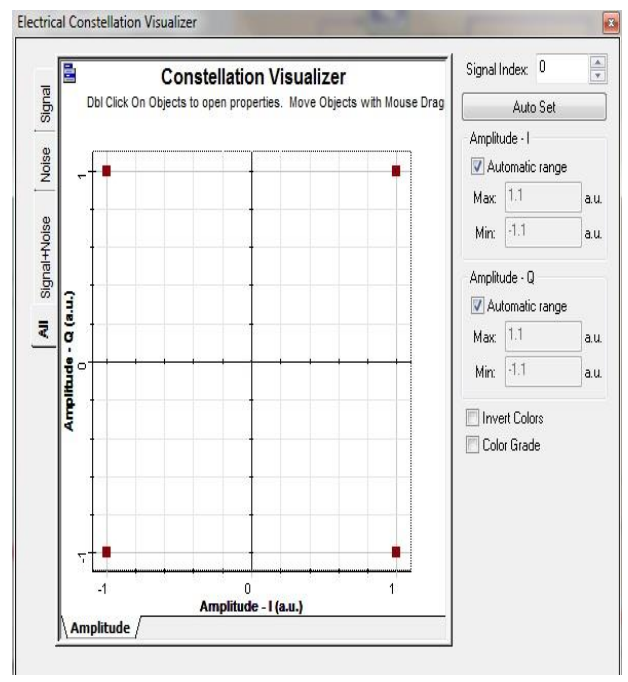

Figure 3. Transmitted Signal Constellation Diagram FOR 4 QAM at the RF OFDM Transmitter 

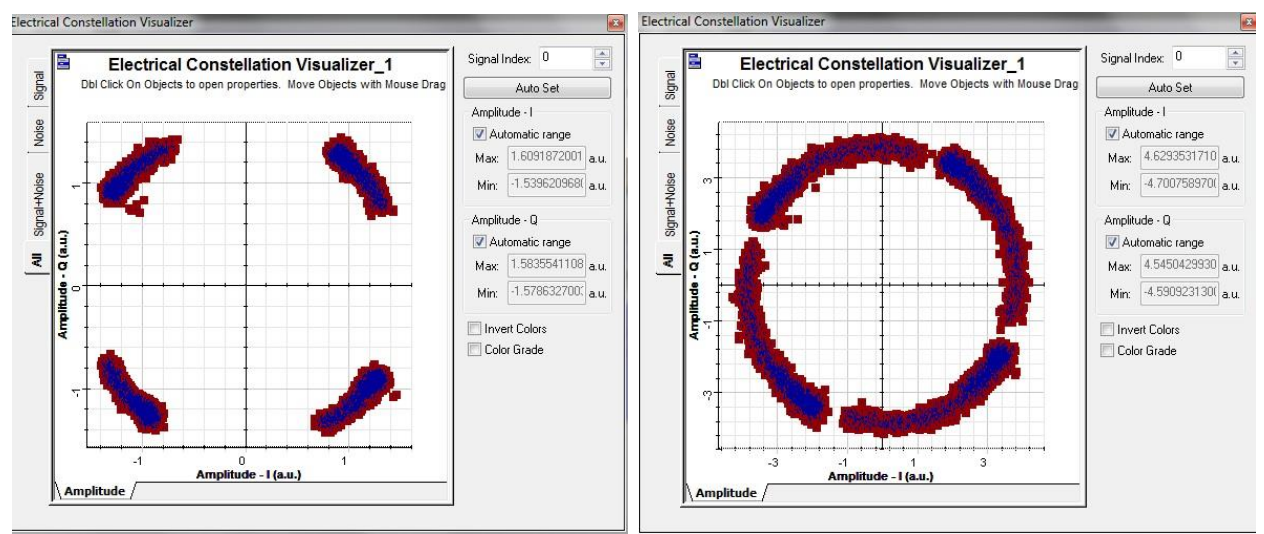

Figure 4. Received Signal Constellation Diagram for 4 QAM at the RF OFDM Receiver after $100 \mathrm{Km}$ and $300 \mathrm{~km}$ SMF
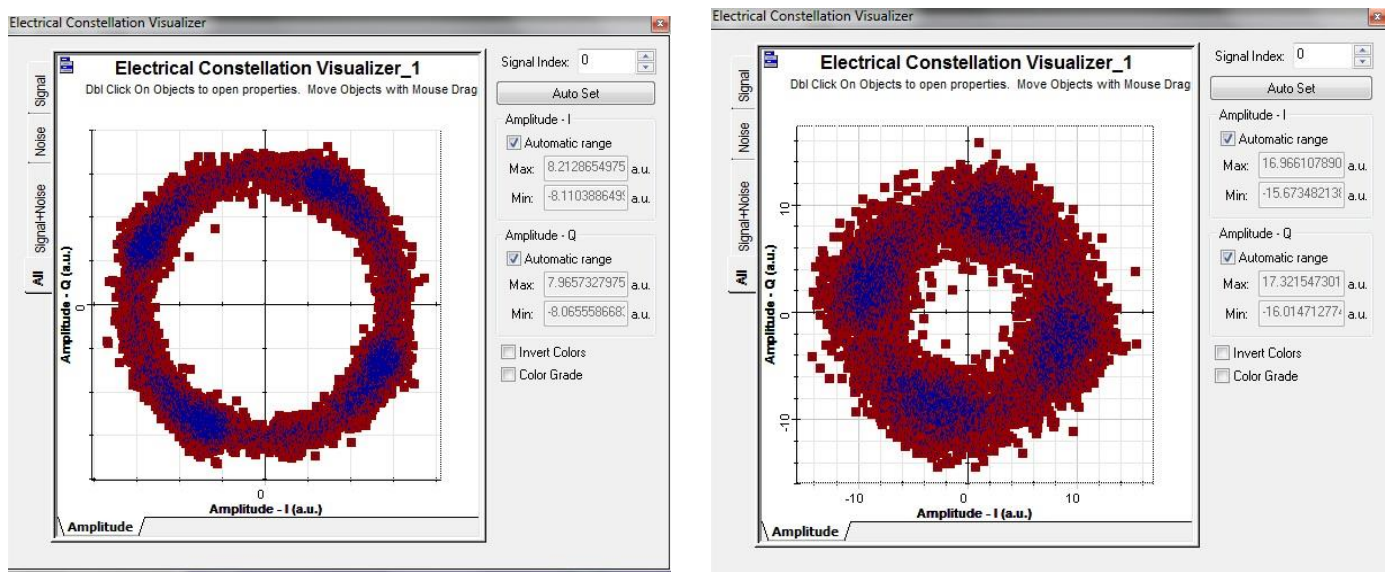

\section{Figure 5. Received Signal Constellation Diagram for 4 QAM at the RF OFDM Receiver after $400 \mathrm{Km}$ and $500 \mathrm{~km}$ SMF}

After propagating through $100 \mathrm{~km}$ SMF 28 fiber, the recovered signal constellation diagram at the RF OFDM receiver is shown below. For transmission of an optical OFDM signal over a long length of fiber, electronic dispersion compensation is necessary. As an example, a 10 Gbps coherent 4-QAM OFDM is transmitted through a $300 \mathrm{~km}$ SMF 28 fiber link. The received signal constellation diagram without electronic dispersion compensation is presented here. It is clearly shown that the signal is corrupted. The signal started broadening when the length of the fiber exceeded $100 \mathrm{~km}$. This broadening occurred in the signal due to the positive dispersion in the SMF. The CD is a major issue in the SMF when the signal travels for a long distance. It can affect the quality of the received signal. The blue dots describe the noise that comes from the laser diode and the red dots represent the signal. 


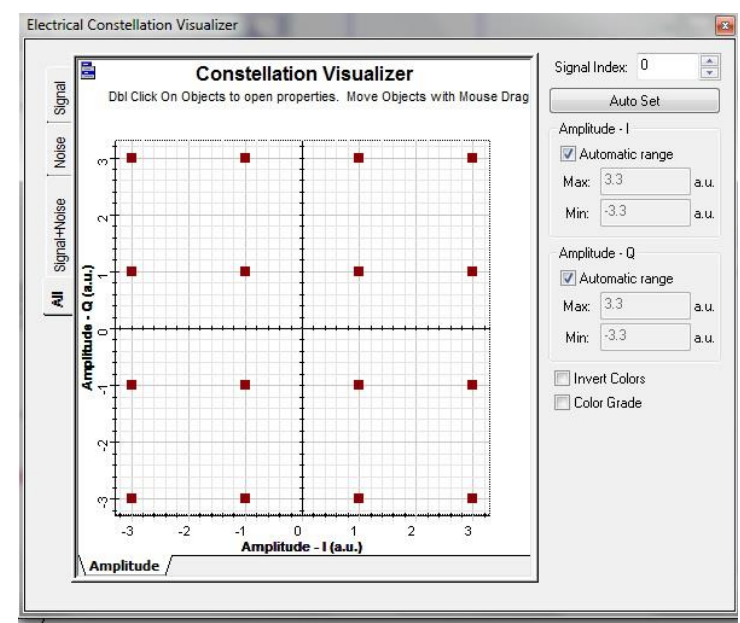

Figure 6. Transmitted Signal Constellation Diagram FOR 4 QAM at the RF OFDM Transmitter
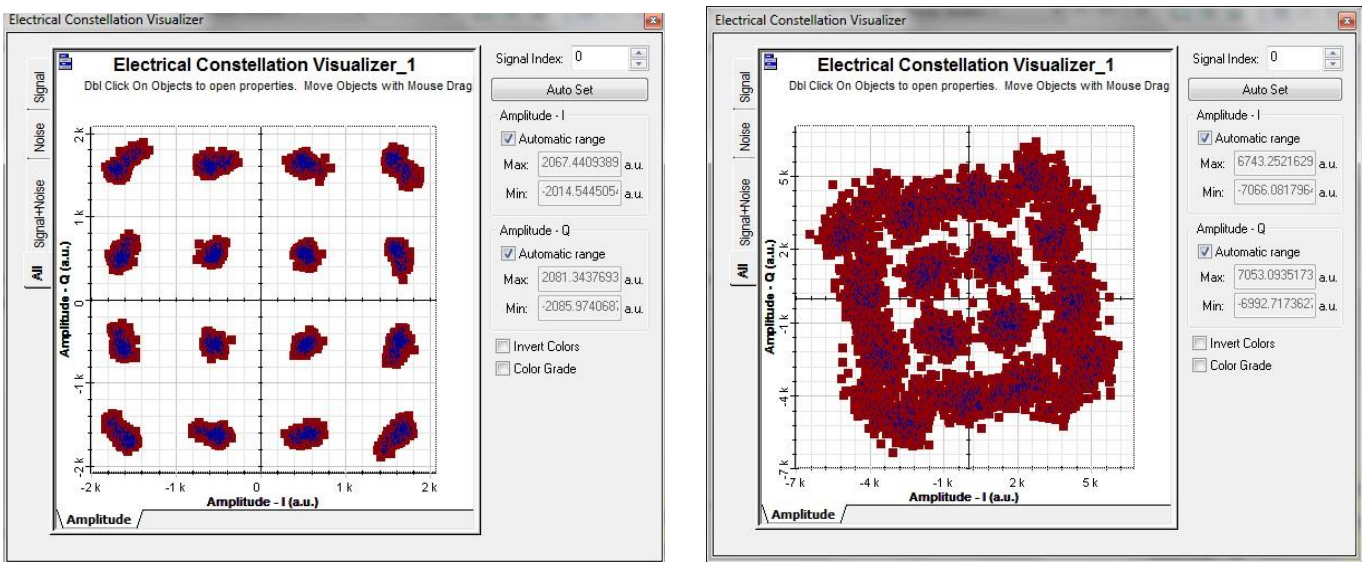

Figure 7. Received Signal Constellation Diagram for 4 QAM at the RF OFDM Receiver after $100 \mathrm{Km}$ and $300 \mathrm{~km}$ SMF
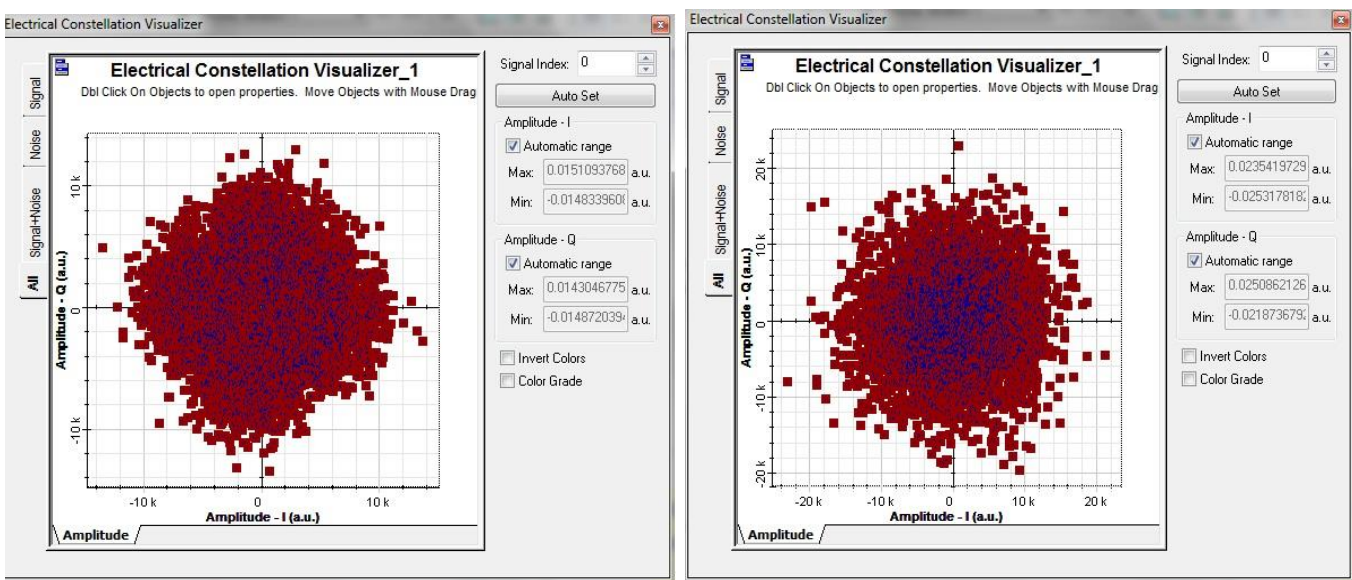

Figure 8. Received Signal Constellation Diagram for 4 QAM at the RF OFDM Receiver after $400 \mathrm{Km}$ and $500 \mathrm{~km}$ SMF

As an example, a $10 \mathrm{Gbps}$ coherent $16-\mathrm{QAM}$ OFDM is transmitted through a $300 \mathrm{~km}$ SMF 28 fiber link. The received signal constellation diagram without electronic dispersion compensation is presented here. It is clearly shown that the signal is corrupted. 
The signal started broadening when the length of the fiber exceeded $100 \mathrm{~km}$. This broadening occurred in the signal due to the positive dispersion in the SMF. The CD is a major issue in the SMF when the signal travels for a long distance. It can affect the quality of the received signal. The blue dots describe the noise that comes from the laser diode and the red dots represent the signal.

\section{Conclusion}

In the proposed architecture, we studied the performance of OFDM-PON downstream coherent detection OFDM using the constellation diagrams of transmitted and received signal that shows the reasonably good performance. Then we have studied the performance of downstream coherent detection m-QAM mapped OFDM by calculating the distance reach of different m-QAM mapped OFDM technique at $10 \mathrm{Gbps}$.This paper has evaluated the performance of coherent optical OFDM system including the effect of fiber nonlinearity. The system is designed using single OFDM signals of $10 \mathrm{~Gb} / \mathrm{s}$ data rate transfer up to $500 \mathrm{~km}$ standard SMF. The result indicates that CO-OFDM system has high spectral efficiency as compared to conventional QPSK Direct detection OFDM system at higher data rates. Thus, for higher data rates and long haul communication CO-OFDM is a better technique as compared to the conventional direct detection (DD) OFDM modulation technique.

\section{References}

[1] G. K. Chang, A. Chowdhury, Z. Jia and H. C. Chien, "Key technologies of WDMPON for future converged optical broadband access networks", IEEE/OSA Journal Opt. Commun. Netw, vol. 1, no. 4, (2009), pp. C35-C50.

[2] S. J. Park, C. H. Lee, K. T. Jeong, H. J. Park, J. G. Ahn and K. H. Song, "Fiber-tothe- home services based on wavelength-division-multiplexing passive optical network", Journal Lightw. Technol, vol. 22, no. 11 , (2004), pp. 2582-2591.

[3] L. G. Kazovsky, W. T. Shaw, D. Gutierrez, N. Cheng and S. W. Wong, "Next generation optical access networks", Jounral Lightw. Technol, vol. 25, no. 11, (2007), pp. 3428-3442.

[4] http://fsanweb.com/archives/category/ next-generation-pon-task-group-ng-pon.

[5] J. I. Kani, F. Bourgart, A. Cui, A. Rafel, M. Campbell, R. Davey and S. Rodrigues, "Next-generation PON-Part I: Technology roadmap and general requirements", IEEE Commun. Mag, vol. 47, no. 11, (2009), pp. 43-49.

[6] I. B. Djordjevic and B. Vasic, "Orthogonal frequency division multiplexing for highspeed optical transmission", Optics Express, vol. 14, no. 9, (2006), pp. 3767-3775.

[7] A. J. Lowery and J. Armstrong, "Orthogonal-frequency-division multiplexing for dispersion compensation of long-haul optical systems", Optics Express, vol. 4, no. 6, (2006), pp. 2079-2084.

[8] P. L. Tien, Y. M. Lin and M. C. Yuang, "A Novel OFDMA-PON Architecture toward Seamless Broadband and Wireless Integration", OFC, paper OMV2, (2009), pp. 1-3.

[9] C. W. Chow, C. H. Yeh and C. H. Wang, "Signal Remodulation of OFDM-QAM for Long Reach Carrier Distributed Passive Optical Networks", IEEE PhotonicsTechnology Letters, vol. 21, no. 11, (2009), pp. 715-717.

[10] V. Tarokh, "New Directions in Wireless Communications Research", US, Springer, (2014).

[11] W. O. Popoola, Z. Ghassemlooy and B. G. Stewart, "Pilot-Assisted PAPR Reduction Technique for Optical OFDM Communication Systems", Journal Lightw. Technol, vol. 32, (2014), pp. 1374-1382.

[12] X. Yi, W. Shieh, Y. Ma, Phase noise effects on high spectral efficiency coherent optical OFDM transmission, J. Lightw. Technol. 6 (2008) 1309-1316.

[13] W. Shieh, H. Bao and Y. Tang, "Coherent optical OFDM: theory and design", Opt. Exp, vol. 16, (2008), pp. 841-859.

[14] W. Shieh and X. Yi, "High spectral efficiency coherent optical OFDM", M. Nakazawa, K. Kikuchi, T. Miyazaki (Eds.), High Spectral Density Optical Communication Technologies, Berlin Heidelberg, Springer, (2010), pp. 141-166.

[15] W. Shieh, "High Spectral Efficiency Coherent Optical OFDM for $1 \mathrm{~Tb} / \mathrm{s}$ Ethernet Transport", Optical Fiber Communication Conference and National Fiber Optic Engineers Conference, (2009), OWW1.

[16] K. Kim, J. Lee and J. Jeong, "Transmission performance of all-optical domain orthogonal frequency division multiplexing signals due to fiber nonlinearities for long-reach PON applications", Opt. Fiber Technol, vol. 18, no. 3, (2012), pp. 140-145. 